\title{
Measurement of global and local resonance terms
}

\author{
R. Tomás, M. Bai, R. Calaga, and W. Fischer \\ Brookhaven National Laboratory, Upton, New York 11973, USA
}

A. Franchi and G. Rumolo

GSI, Planckstrasse 1, 64291 Darmstadt, Germany

(Received 29 October 2004; published 22 February 2005)

\begin{abstract}
Recently, resonance driving terms were successfully measured in the CERN SPS and the BNL RHIC from the Fourier spectrum of beam position monitor (BPM) data. Based on these measurements a new analysis has been derived to extract truly local observables from BPM data. These local observables are called local resonance terms since they share some similarities with the global resonance terms. In this paper we derive these local terms analytically and present experimental measurements of sextupolar global and local resonance terms in RHIC. Nondestructive measurements of these terms using ac dipoles are also presented.
\end{abstract}

DOI: 10.1103/PhysRevSTAB.8.024001

PACS numbers: $29.20 .-\mathrm{c}$

\section{INTRODUCTION AND THEORY}

In [1] normal form and Lie algebra techniques were used to describe the motion of a particle confined in an accelerator in the presence of nonlinearities. The particle position $x_{1}$ as a function of the turn number $N$ at a certain location (indexed by 1) was given the following form,

$$
\begin{aligned}
x_{1}(N)= & \sqrt{\beta_{x 1}} \operatorname{Re}\left\{\sqrt{2 I_{x}} e^{i\left(2 \pi \nu_{x} N+\psi_{x_{1}}\right)}\right. \\
& -2 i \sum_{j k l m} j f_{j k l m}^{(1)}\left(2 I_{x}\right)^{(j+k-1) / 2}\left(2 I_{y}\right)^{(l+m) / 2} \\
& \left.\times e^{i\left[(1-j+k)\left(2 \pi \nu_{x} N+\psi_{x_{1}}\right)+(m-l)\left(2 \pi \nu_{y} N+\psi_{y_{1}}\right)\right]}\right\},
\end{aligned}
$$

where $I_{x, y}$ are the horizontal and vertical actions, $\nu_{x, y}$ are the tunes, $\psi_{x_{1}, y_{1}}$ are the initial phases, and $f_{j k l m}^{(1)}$ are the generating function terms. The generating function terms are directly related to the Hamiltonian terms $h_{j k l m}^{(1)}$ as follows:

$$
f_{j k l m}^{(1)}=\frac{h_{j k l m}^{(1)}}{1-e^{-i 2 \pi\left[(j-k) Q_{x}+(l-m) Q_{y}\right]}} .
$$

In [2] it was found that these terms experience a characteristic variation around the accelerator lattice: their amplitude remains constant in sections free of multipoles and shows abrupt jumps at the locations of these sources. The analytical expression describing these abrupt changes is given by

$$
\begin{aligned}
f_{j k l m}^{(2)}= & e^{-i\left[(k-j) \Delta \phi_{x}+(m-l) \Delta \phi_{y}\right]}\left[f_{j k l m}^{(1)}\right. \\
& \left.-\sum_{q=1}^{n} e^{i(k-j) \phi_{x q}+i(m-l) \phi_{y q}} h_{q_{j k l m}}\right]
\end{aligned}
$$

where $f_{j k l m}^{(2)}$ is the generating term at a second location, $\Delta \phi_{x, y}$ are the horizontal and vertical phase advances between the two locations, the summation extends only over the multipoles placed between the two locations, $\phi_{x q, y q}$ are the phase advances between the first location and the $q$ th multipole, and $h_{q_{j k l m}}$ are real quantities proportional to the strength of the $q$ th multipole and to the product $\beta_{x q}^{(j+k) / 2} \beta_{y q}^{(l+m) / 2}$; see [3] for a more detailed expression.

In [2] the measurement of amplitudes and phases of generating function terms was successfully achieved at two accelerators: the CERN SPS and the BNL RHIC. This measurement together with Eq. (3) opens the possibility of measuring magnet strengths. Indeed, if there is only one multipole between the two locations 1 and 2, its strength can be directly inferred knowing the betatron functions. Nevertheless there are two limitations to this approach: (1) The existence of several multipoles between the two locations avoids the measurement of particular strengths. When this is the case an integrated strength is obtained, namely the summation in Eq. (3). (2) The measurement of $f_{j k l m}$ at one location needs two beam position monitors (BPMs) with a phase advance different from $0^{\circ}$ and $180^{\circ}$, for the momentum reconstruction. If nonlinearities exist between these two BPMs, $f_{j k l m}$ can be measured only up to an error on the order of the strength of these nonlinearities. This error is usually smaller than $f_{j k l m}$ but would not be negligible when measuring magnet strengths.

The first limitation is unavoidable given the BPM configuration. The second one is overcome by adopting another approach using three BPMs. This new method follows.

\section{Three BPM method}

Assume that Fig. 1 represents the BPM and multipole configuration of a certain segment of an accelerator. The 


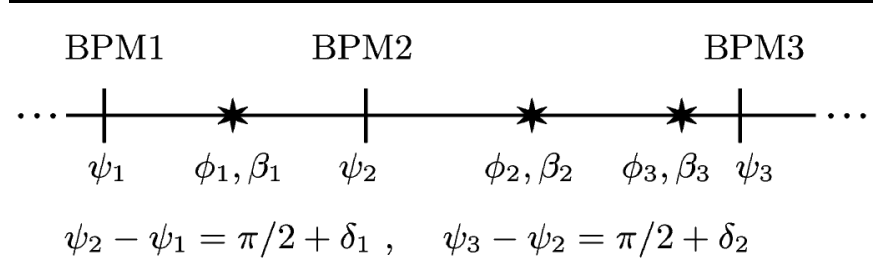

FIG. 1. Segment of an accelerator lattice. BPMs and multipoles are shown with their relevant Twiss parameters.

relevant beta functions $(\beta)$ and phase advances ( $\phi$ for magnets and $\psi$ for BPMs) are shown in the figure.

For convenience $\delta_{1}$ and $\delta_{2}$ have been introduced as the deviation from $90^{\circ}$ of the phase advance between consecutive BPMs. The only constraint imposed on these quantities is that $\cos \left(\delta_{1,2}\right) \neq 0$. This is to avoid zero denominators in the equations below. A local observable is constructed from the BPMs' turn-by-turn signals as follows,

$$
\chi(N)=\frac{\hat{x}_{1}(N)}{\cos \delta_{1}}+\hat{x}_{2}(N)\left(\tan \delta_{1}+\tan \delta_{2}\right)+\frac{\hat{x}_{3}(N)}{\cos \delta_{2}},
$$

where the hat means that the signal has been normalized to the amplitude of the fundamental betatron oscillation. These amplitudes and the phase advances $\delta_{1}$ and $\delta_{2}$ are obtained from the Fourier transform of the signals. For an ideal uncoupled linear machine, $\chi(N)=0$ for any $N$ and for any set of three BPMs. Furthermore, in the presence of multipoles distributed around the ring, $\chi(N)$ only depends on those nonlinearities placed between the three BPMs. The analytical proof of these statements and the following expressions is given in Appendix A. The equation that relates $\chi(N)$ and the local sources is given by

$$
\begin{aligned}
\chi(N)= & 4 \sum_{j>k, l>m} j\left|\chi_{j k l m}\right|\left(2 I_{x}\right)^{(j+k-2) / 2}\left(2 I_{y}\right)^{(l+m) / 2} \\
& \times \cos \left(\left((1-j+k) \nu_{x}+(m-l) \nu_{y}\right) 2 \pi N+\psi_{j k l m}\right),
\end{aligned}
$$

where the local terms $\chi_{j k l m}$ and the phases $\psi_{j k l m}$ are defined as

$$
\begin{aligned}
& \chi_{j k l m}=\sum_{q=1}^{n^{\prime}} e^{i\left[(1-j+k) \phi_{x q}+(m-l) \phi_{y q}\right]} \operatorname{SEN}\left(\phi_{x q}\right) h_{q_{j k l m}}, \\
& \psi_{j k l m}=(1-j+k) \psi_{x 1}+(m-l) \psi_{y 1}+\arg \left(\chi_{j k l m}\right),
\end{aligned}
$$

where the summation extends over the multipoles in between the three BPMs, $h_{q_{j k l m}}$ are the quantities proportional to the strengths already introduced in Eq. (3), and the function $\operatorname{SEN}\left(\phi_{x q}\right)$ is defined as

$$
\begin{cases}\sin \phi_{x q} \sqrt{1+\tan ^{2} \delta_{1}} & \text { if } \phi_{x q}<\psi_{2}-\psi_{1} \\ \sin \left(\phi_{x q}-\delta_{1}-\delta_{2}\right) \sqrt{1+\tan ^{2} \delta_{2}} & \text { if } \phi_{x q}>\psi_{2}-\psi_{1} .\end{cases}
$$

Note that the above expressions largely simplify when $\delta_{1}=\delta_{2}=0$, giving $\chi(N)=\hat{x}_{1}(N)+\hat{x}_{3}(N)$ and $\operatorname{SEN}\left(\phi_{x q}\right)=\sin \phi_{x q}$. This situation corresponds to two BPMs separated by exactly $180^{\circ}$.

We have constructed a local observable $\chi(N)$ that depends both on local magnet strengths and the distribution of the three BPMs. The Fourier coefficients of this observable provide the local terms $\chi_{j k l m}$, which are similar to the Hamiltonian terms but strictly local. There is a fundamental difference between the local and the global resonance terms: the global terms are related to only one resonance while the local terms are related to two resonances; see Appendix A 1. This is due to the fact that $\chi(N)$ is a real quantity as opposed to the traditional complex variable used to derive the global resonance terms. As a consequence, the numbers of independent spectral lines and local terms are reduced to a half. In accordance, the summation of Eq. (5) extends only over $j>k$ and $l>m$. The measurement of the local terms represents a means of finding lattice imperfections or unexpected multipoles in an accelerator.

\section{RHIC MODEL}

In order to compare results from the measurements of sextupolar components to predictions, a MADX model of the RHIC yellow injection lattice has been constructed. The interaction regions (IRs) have been modeled as described in [4] using the corresponding magnet measurements. Some dipoles in the IRs do not have magnetic measurements. No sextupolar components have been assumed for them. The arcs contain the chromaticity sextupoles and the sextupolar components of the superconducting dipoles. The arc dipoles have been sliced into eight slices and the corresponding sextupolar multipoles have been placed in between.

\section{RHIC EXPERIMENTS}

During 2004 RHIC gold operation experiments to measure magnet strengths from BPM data were carried out in a similar way as in [5]. Transverse betatron oscillations were excited either by injecting off orbit or by driving forced oscillations with the aid of an ac dipole. 1024 turn-by-turn BPM data were recorded after every transverse excitation. All experiments were performed at injection energy. The tunes were moved closer to the third order resonance to enhance the sextupolar resonances, $Q_{x}=0.31$ and $Q_{y}=$ 0.22 . Chromaticities were $Q_{x, y}^{\prime} \approx-2$ units, where the prime denotes the derivative with respect to the relative momentum deviation. For the presented measurements no IR correction circuits were used.

Prior to the data analysis the malfunctioning BPMs were removed as reported in [6]. Yet a new failure mode of the BPM system had to be pursued: few BPMs report on a different turn number than the rest. To find these faulty BPMs the phase advance between consecutive BPMs as 
measured from the Fourier transform is compared to that predicted by the model. Those few BPMs having a larger deviation from the model than the rest were rejected.

\section{A. Measurement of $\chi_{\mathbf{3 0 0 0}}$ from kick data}

The measurement of $\chi_{3000}$ is performed in a similar way to that of $f_{3000}$ as described in [2]. It is interesting to note that the term $\chi_{1200}$ does not exist in Eq. (5) as opposed to $f_{1200}$ that is also measurable. The contribution of this local term to $\chi(N)$ is given by

$$
12\left|\chi_{3000}\right|\left(2 I_{x}\right)^{1 / 2} \cos \left(4 \pi \nu_{x} N+\psi_{3000}\right) .
$$

To measure $\left|\chi_{3000}\right|$ a line constrained to go trough the origin is fitted to the amplitude of the spectral line with frequency $-2 \nu_{x}[$ line $(-2,0)]$ from the Fourier spectrum of $\chi(N)$ versus $\sqrt{2 I_{x}}$. Using the line $(2,0)$ would give identical results since $\chi(N)$ is a real quantity. An example of this fit is shown in Fig. 2 for a particular set of three BPMs. The quality of the data is poor at the lower oscillation amplitudes since we are measuring a small quantity. The measurement of even smaller quantities would need either larger oscillation amplitudes or the analysis of longer samples of undecohered data, obtained, for instance, with an ac dipole as will be explained below. $\sqrt{2 I_{x}}$ is measured from the peak-to-peak amplitude of the BPM readings and from a measurement of the beta functions throughout the ring. The measurement of these functions relies on the betas predicted from the RHIC model. The final error of $\sqrt{2 I_{x}}$ is shown in the figure. The effect of beam decoherence has to be taken into account as described in [2]. If the centroid oscillations are damped due to amplitude detuning, the line $(n, 0)$ is reduced by a factor of $|n|$. The line $(-2,0)$ is therefore reduced by a factor of 2 . $\left|\chi_{3000}\right|$ is given by one sixth [from Eq. (8) and taking into account the factor of 2 from decoherence].

The measurement of $\left|\chi_{3000}\right|$ around the RHIC yellow ring is shown in the central plot of Fig. 3 with a comparison

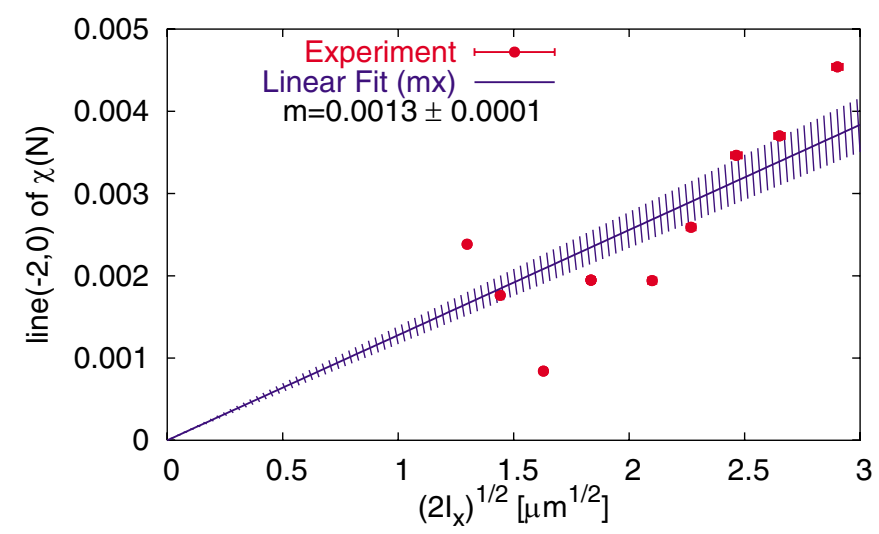

FIG. 2. (Color) Amplitude of the spectral line with frequency $-2 \nu_{x}$ [line $\left.(-2,0)\right]$ from the Fourier spectrum of $\chi(N)$ versus $\sqrt{2 I_{x}}$.

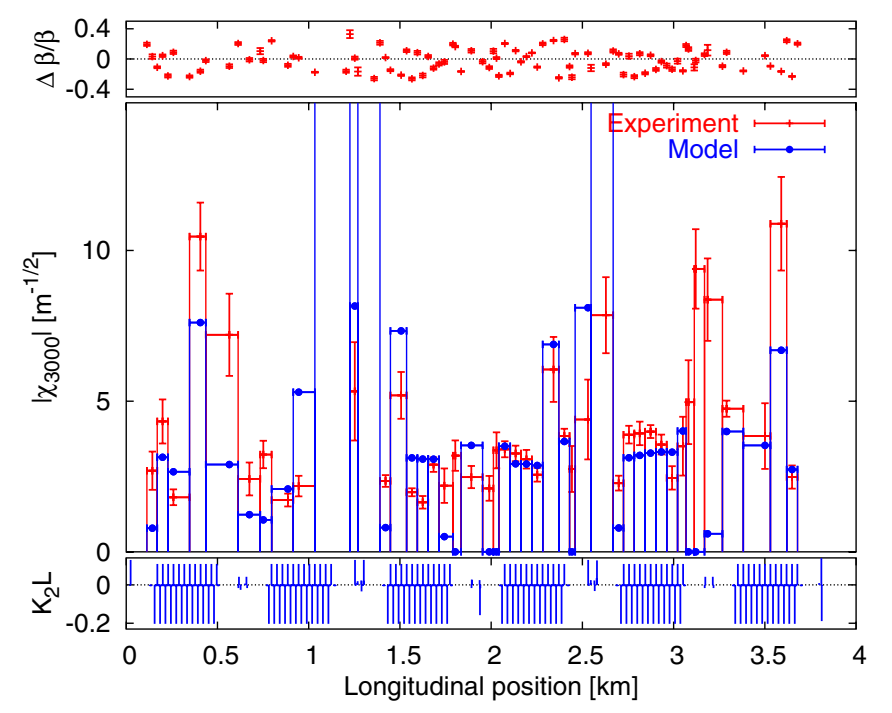

FIG. 3. (Color) Measurement of $\left|\chi_{3000}\right|$ from kick data. The top plot shows the beta beating. The middle plot shows $\left|\chi_{3000}\right|$ around the ring with a comparison to the model. The bottom plot shows the sextupolar components of the ring.

to the model. The horizontal error bars are used to delimit the segment of the lattice occupied by the three BPMs (vertical lines are also used at the edges of the segment). The central dot corresponds to the location of the middle BPM. Model and experiment show good agreement in the arcs. Discrepancies arise in the IRs partly due to the fact that the model is not complete in these regions. The top plot of the figure shows the measured beta beating with respect to the model. The bottom plot shows the distribution of the sextupolar components used in the model.

\section{B. Measurements using an ac dipole}

An ac dipole drives transverse beam oscillations at a frequency close to the betatron tune. The nondestructive measurement of resonance driving terms using an ac dipole was proposed in [7]. The main finding of this paper was that the resonance driving terms in the presence of an ac dipole, $f_{j k l m}^{\prime}$, differ from the natural resonance driving terms $f_{j k l m}$ in a quantity that increases with the separation of the driving and the betatron tunes.

$f_{j k l m}^{\prime}$ has been measured for the first time around the RHIC yellow lattice. The betatron horizontal tune was $Q_{x}=0.31$ and the driving tune of the ac dipole was set to $Q_{D}=0.32$. The ac dipole was adiabatically switched on and off at various excitation amplitudes in order to be able to measure $f_{j k l m}^{\prime}$ as proposed in [7]. The measurement is shown in Fig. 4 together with a prediction from the model. The horizontal error bars delimit the locations of the two BPMs. The few points that show a discrepancy have a large horizontal error bar. This large separation of the BPMs compromises the reconstruction of the momentum, introducing an error of the order of the nonlinearities within this region, as shown in Eq. (A6). 


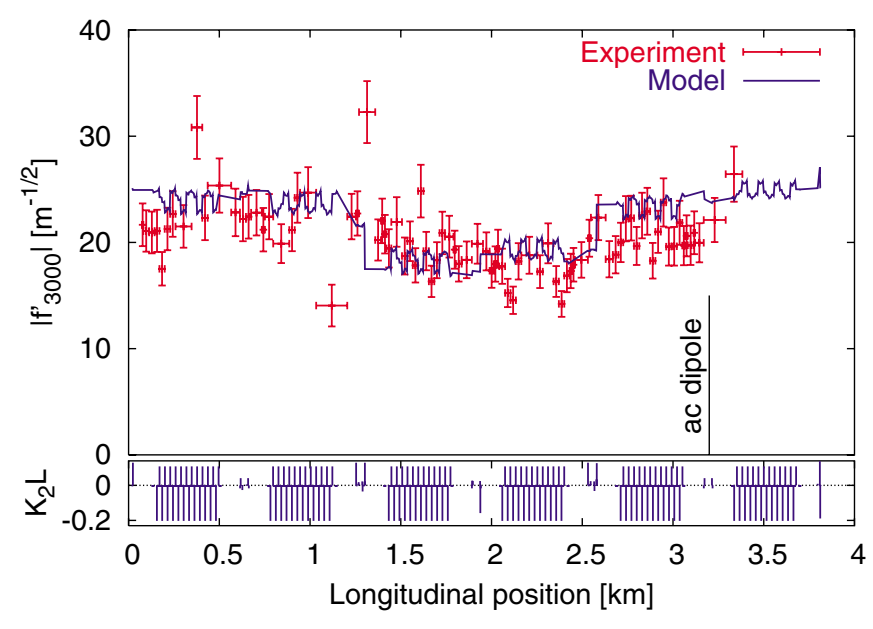

FIG. 4. (Color) Measurement of $\left|f_{3000}^{\prime}\right|$ with an ac dipole. The bottom plot shows the sextupolar components of the ring.

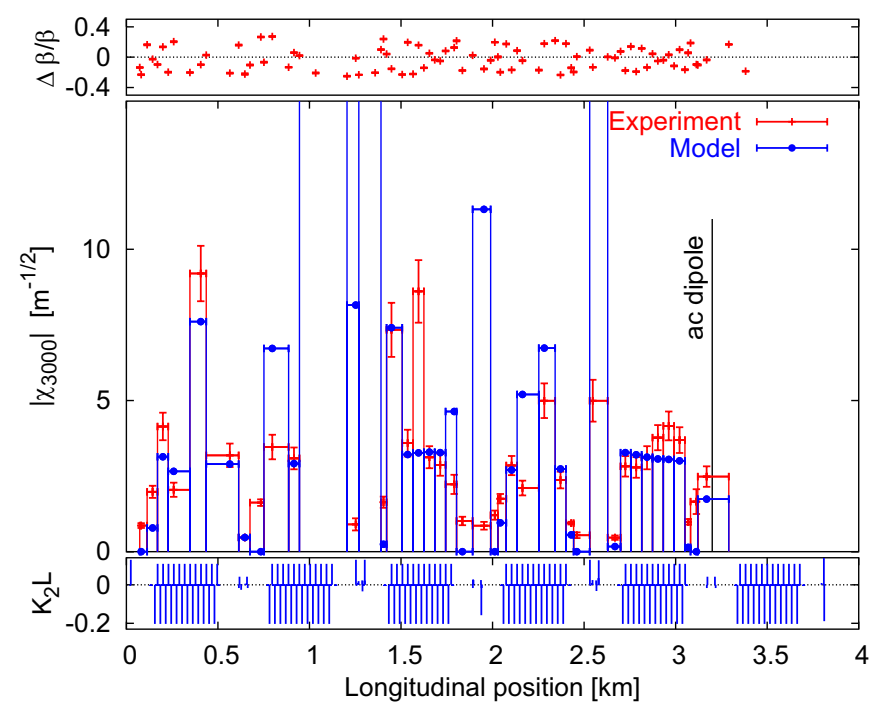

FIG. 5. (Color) Measurement of $\left|\chi_{3000}\right|$ with an ac dipole. The bottom plot shows the sextupolar components of the ring.

In Appendix B it is demonstrated that under certain assumptions the amplitude of the measured local resonance terms is independent of the nature of the beam oscillations, i.e., free oscillations or driven by using an ac dipole. Consequently, we have measured $\left|\chi_{3000}\right|$ proceeding in the same way as in the previous section but using ac dipole data. This method has the advantage that no decoherence factor has to be taken into account since the oscillations are not damped. The result is shown in Fig. 5. The agreement is similar or better than for the kick case. This demonstrates the feasibility of this kind of measurement.

\section{CONCLUSION}

The BPM-based observables $\chi(N)$ and $\chi_{j k l m}$ have been defined. Up to first order in the nonlinearities of the lattice these observables only depend on the sources placed in between the three BPMs used. The sextupolar local term $\chi_{3000}$ has been measured around RHIC from both free oscillation data and driven oscillation data by using an ac dipole. The sextupolar generating function term has also been measured using an ac dipole for the first time.

\section{ACKNOWLEDGMENTS}

The authors thank F. Pilat for collaboration in the experiments and J. van Zeijts for help with the RHIC on-line model. This work has been performed under the auspices of the U.S. Department of Energy.

\section{APPENDIX A: PROOF OF THE PROPERTIES OF $\chi(N)$}

$\chi(N)$ is defined from the turn-by-turn horizontal beam positions at three arbitrary locations as

$$
\chi(N)=\frac{\hat{x}_{1}(N)}{\cos \delta_{1}}+\hat{x}_{2}(N)\left(\tan \delta_{1}+\tan \delta_{2}\right)+\frac{\hat{x}_{3}(N)}{\cos \delta_{2}} .
$$

For convenience $\chi(N)$ is expressed in terms of complex variables as

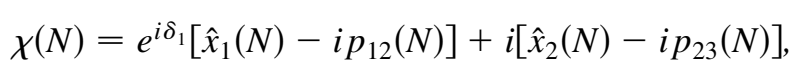

where $p_{r t}(N)$ is the reconstructed momentum from the coordinates $\hat{x}_{r}(N)$ and $\hat{x}_{t}(N)$ defined as

$$
p_{r t}(N)=\left(\hat{x}_{t}(N)+\hat{x}_{r}(N) \sin \delta_{r}\right) / \cos \delta_{r} .
$$

We aim to express $\chi(N)$ as a function of the sources located in between the three BPMs up to first order. To this aim Eqs. (1) and (3) are used to perform the appropriate substitutions in three main steps: (1) computation of the reconstructed momentum, (2) computation of the complex variable, and (3) computation of $\chi(N)$. In the following each step is reported in one section.

\section{Computation of $p_{12}$}

Using Eq. (1) the reconstructed momentum of Eq. (A3), for instance $p_{12}$, is given by

$$
\begin{aligned}
p_{12}= & \tan \delta_{1} \operatorname{Re}\left\{e^{i\left(2 \pi \nu_{x} N+\psi_{x_{1}}\right)}-2 i \sum_{j k l m} j f_{j k l m}^{(1)}\left(2 I_{x}\right)^{(j+k-2) / 2}\left(2 I_{y}\right)^{(l+m) / 2} e^{i\left[(1-j+k)\left(2 \pi \nu_{x} N+\psi_{x_{1}}\right)+(m-l)\left(2 \pi \nu_{y} N+\psi_{y_{1}}\right)\right]}\right\} \\
& +\frac{1}{\cos \delta_{1}} \operatorname{Re}\left\{e^{i\left(2 \pi \nu_{x} N+\psi_{x_{2}}\right)}-2 i \sum_{j k l m} j f_{j k l m}^{(2)}\left(2 I_{x}\right)^{(j+k-2) / 2}\left(2 I_{y}\right)^{(l+m) / 2} e^{i\left[(1-j+k)\left(2 \pi \nu_{x} N+\psi_{x_{2}}\right)+(m-l)\left(2 \pi \nu_{y} N+\psi_{y_{2}}\right)\right]}\right\} .
\end{aligned}
$$


After some algebra the reconstructed momentum yields

$$
\begin{aligned}
p_{12}= & \cos \left(2 \pi \nu_{x} N+\psi_{x_{1}}+\pi / 2\right)-\frac{2}{\cos \delta_{1}} \operatorname{Re}\left\{i \sum_{j k l m} j\left[f_{j k l m}^{(1)} \sin \delta_{1}+f_{j k l m}^{(2)} e^{i(1-j+k)\left(\pi / 2+\delta_{1}\right)+i(m-l)\left(\psi_{y_{2}}-\psi_{y_{1}}\right)}\right]\right. \\
& \left.\times\left(2 I_{x}\right)^{(j+k-2) / 2}\left(2 I_{y}\right)^{(l+m) / 2} e^{i\left[(1-j+k)\left(2 \pi \nu_{x} N+\psi_{x_{1}}\right)+(m-l)\left(2 \pi \nu_{y} N+\psi_{y_{1}}\right)\right]}\right\} .
\end{aligned}
$$

Using Eq. (3) the quantity between the square brackets of this equation is expressed as

$$
f_{j k l m}^{(1)} \sin \delta_{1}+f_{j k l m}^{(2)} e^{i(1-j+k)\left(\pi / 2+\delta_{1}\right)+i(m-l)\left(\psi_{y_{2}}-\psi_{y_{1}}\right)}=f_{j k l m}^{(1)} \sin \delta_{1}+\left[f_{j k l m}^{(1)}-\sum_{q=1}^{n} e^{i(k-j) \phi_{x q}+i(m-l) \phi_{y q}} h_{q_{j k l m}}\right] e^{i\left(\pi / 2+\delta_{1}\right)} ;
$$

$p_{12}$ is then given by

$$
\begin{aligned}
p_{12}= & \cos \left(2 \pi \nu_{x} N+\psi_{x_{1}}+\pi / 2\right)+2 \sum_{j k l m}\left(2 I_{y}\right)^{(l+m) / 2} \operatorname{Re}\left\{j\left[f_{j k l m}^{(1)}-\frac{e^{i \delta_{1}}}{\cos \delta_{1}} \sum_{q=1}^{n} e^{i(k-j) \phi_{x q}+i(m-l) \phi_{y q}} h_{q_{j k l m}}\right]\right. \\
& \left.\times\left(2 I_{x}\right)^{(j+k-2) / 2} e^{i\left[(1-j+k)\left(2 \pi \nu_{x} N+\psi_{x_{1}}\right)+(m-l)\left(2 \pi \nu_{y} N+\psi_{y_{1}}\right)\right]}\right\} .
\end{aligned}
$$

This reconstructed momentum differs from the real momentum at the first location only by the term containing the summation over the local sources in between the square brackets. Therefore this expression can also be used to estimate the error in the reconstruction of the momentum from two BPMs when nonlinear sources are placed in between. But this "error" is indeed the part of the equation we are most interested since it is truly local. An equivalent expression is obtained for $p_{23}$ by replacing the indexes in this equation. The summation over the sources would expand over the sources placed between the second and third locations, say there are $n^{\prime}$ sources.

\section{Computation of the complex variable}

The next step is to compute the complex variable defined as $\hat{x}_{1}(N)-i p_{12}(N)$, which by using Eqs. (1) and (A6) takes the form

$$
\begin{aligned}
\hat{x}_{1}(N)-i p_{12}(N)= & e^{i\left(2 \pi \nu_{x} N+\psi_{x_{1}}\right)}-2 i \sum_{j k l m} j f_{j k l m}^{(1)}\left(2 I_{y}\right)^{(l+m) / 2}\left(2 I_{x}\right)^{(j+k-2) / 2} e^{i\left[(1-j+k)\left(2 \pi \nu_{x} N+\psi_{x_{1}}\right)+(m-l)\left(2 \pi \nu_{y} N+\psi_{y_{1}}\right)\right]} \\
& +i \operatorname{Re}\left\{-2 i \sum_{j k l m} \sum_{q=1}^{n} j e^{i(k-j)\left(\phi_{x q}-\pi / 2\right)+i(m-l)\left(\phi_{y q}-\Delta \phi_{y}\right)} h_{q_{j k l m}}\left(1+i \tan \delta_{1}\right)\left(2 I_{x}\right)^{(j+k-2) / 2}\right. \\
& \left.\times\left(2 I_{y}\right)^{(l+m) / 2} e^{i\left[(1-j+k)\left(2 \pi \nu_{x} N+\psi_{x_{2}}\right)+(m-l)\left(2 \pi \nu_{y} N+\psi_{y_{2}}\right)\right]}\right\} .
\end{aligned}
$$

Using that $\operatorname{Re}\{a\}=\left(a+a^{*}\right) / 2$ for any complex number $a$, the complex variable can be expressed in the following way:

$$
\hat{x}_{1}(N)-i p_{12}(N)=e^{i\left(2 \pi \nu_{x} N+\psi_{x_{1}}\right)}-2 i \sum_{j k l m} j \bar{f}_{j k l m}^{(1)}\left(2 I_{x}\right)^{(j+k-2) / 2}\left(2 I_{y}\right)^{(l+m) / 2} e^{i\left[(1-j+k)\left(2 \pi \nu_{x} N+\psi_{x_{1}}\right)+(m-l)\left(2 \pi \nu_{y} N+\psi_{y_{1}}\right)\right]},
$$

where $\bar{f}_{j k l m}^{(1)}$ is given by

$$
\begin{aligned}
f_{j k l m}^{(1)} & +e^{i\left[(1-j+k)\left(\psi_{x_{2}}-\psi_{x_{1}}\right)+(m-l)\left(\psi_{y_{2}}-\psi_{y_{1}}\right)\right]}\left[+\frac{i}{2} \sum_{q=1}^{n} e^{i(k-j)\left(\phi_{x q}-\pi / 2\right)+i(m-l)\left(\phi_{y q}-\Delta \phi_{y}\right)} h_{q_{j k l m}}\left(1+i \tan \delta_{1}\right)\right. \\
& \left.-\frac{i}{2} \sum_{q=1}^{n} \frac{j^{\prime}}{j} e^{-i\left(k^{\prime}-j^{\prime}\right)\left(\phi_{x q}-\pi / 2\right)-i\left(m^{\prime}-l^{\prime}\right)\left(\phi_{y q}-\Delta \phi_{y}\right)} h_{q_{j^{\prime} k^{\prime} l^{\prime} m^{\prime}}}\left(1-i \tan \delta_{1}\right)\right]
\end{aligned}
$$

and the new indexes with the prime are to fulfill

$$
1-j^{\prime}+k^{\prime}=-(1-j+k), \quad m^{\prime}-l^{\prime}=-(m-l), \quad j^{\prime}+k^{\prime}=j+k, \quad m^{\prime}+l^{\prime}=m+l .
$$


Therefore, $m^{\prime}=l, l^{\prime}=m, k^{\prime}=j-1$, and $j^{\prime}=k+1$. The term $h_{q_{j k l m}}$ is proportional to the factor

$$
\frac{(j+k) !}{j ! k !}
$$

By virtue of these relations we have

$$
h_{q_{j^{\prime} k^{\prime} l^{\prime} m^{\prime}}}=\frac{j}{k+1} h_{q_{j k l m}}
$$

Using the above relations and that

$$
1 \pm i \tan \delta_{1}=\sqrt{1+\tan ^{2} \delta_{1}} e^{ \pm i \delta_{1}}
$$

$\bar{f}_{j k l m}^{(1)}$ is expressed as

$$
\bar{f}_{j k l m}^{(1)}=f_{j k l m}^{(1)}-\sqrt{1+\tan ^{2} \delta_{1}} \sum_{q=1}^{n} e^{i(1+k-j) \phi_{x q}+i(m-l) \phi_{y q}} \cos \left(\phi_{x q}-\delta_{1}\right) h_{q_{j k l m}} .
$$

$\bar{f}_{j k l m}^{(1)}$ can be regarded as the generating function term with an error due to the nonlinear sources placed in between the two locations used to reconstruct the momentum. Again, this error is the interesting part of the equation for us. $\bar{f}_{j k l m}^{(2)}$ can be obtained by replacing the index 1 by 2 and including in the summation only the sources placed between the second and the third location.

\section{Computation of $\chi(N)$}

Using Eqs. (A2) and (A8) $\chi(N)$ is expressed as

$$
\begin{aligned}
\chi(N)= & e^{i\left(2 \pi \nu_{x} N+\psi_{x_{1}}+\delta_{1}\right)}-2 i e^{i \delta_{1}} \sum_{j k l m} j \bar{f}_{j k l m}^{(1)}\left(2 I_{y}\right)^{(l+m) / 2}\left(2 I_{x}\right)^{(j+k-2) / 2} e^{i\left[(1-j+k)\left(2 \pi \nu_{x} N+\psi_{x_{1}}\right)+(m-l)\left(2 \pi \nu_{y} N+\psi_{y_{1}}\right)\right]} \\
& +e^{i\left[2 \pi \nu_{x} N+\psi_{x_{2}}+(\pi / 2)\right]}-2 i e^{i(\pi / 2)} \sum_{j k l m} j \bar{f}_{j k l m}^{(2)}\left(2 I_{y}\right)^{(l+m) / 2}\left(2 I_{x}\right)^{(j+k-2) / 2} e^{i\left[(1-j+k)\left(2 \pi \nu_{x} N+\psi_{x_{2}}\right)+(m-l)\left(2 \pi \nu_{y} N+\psi_{y_{2}}\right)\right]}
\end{aligned}
$$

The purely exponential terms cancel out and all the remaining monomials are proportional to the resonance terms,

$\chi(N)=-2 i e^{i \delta_{1}} \sum_{j k l m} j\left(\bar{f}_{j k l m}^{(1)}-\bar{f}_{j k l m}^{(2)} e^{i\left[(k-j)\left(\pi / 2+\delta_{1}\right)+(m-l) \Delta \psi_{y}\right]}\right)\left(2 I_{y}\right)^{(l+m) / 2}\left(2 I_{x}\right)^{(j+k-2) / 2} e^{i\left[(1-j+k)\left(2 \pi \nu_{x} N+\psi_{x_{1}}\right)+(m-l)\left(2 \pi \nu_{y} N+\psi_{y_{1}}\right)\right]}$.

Using Eqs. (A13) and (3) the quantity in between the large brackets is expressed as

$$
\bar{f}_{j k l m}^{(1)}-\bar{f}_{j k l m}^{(2)} e^{i\left[(k-j)\left(\pi / 2+\delta_{1}\right)+(m-l) \Delta \psi_{y}\right]}=i e^{-i \delta_{1}} \sum_{q=1}^{n^{\prime}} e^{i(1+k-j) \phi_{x q}+i(m-l) \phi_{y q}} \operatorname{SEN}\left(\phi_{x q}\right) h_{q_{j k l m}},
$$

where the function SEN was already introduced in Eq. (7). The summation extends now over all the sources placed in between the three BPMs. The resonance driving terms have canceled out and only the contribution from the sources placed in between the BPMs remains. This proves that $\chi(N)$ is a local observable. It is trivial to see that if there are no local sources $\left(h_{q_{j k l m}}=0\right) \chi(N)$ is zero. $\chi(N)$ is then given by 


$$
\chi(N)=2 \sum_{j k l m} \sum_{q=1}^{n^{\prime}} j e^{i(1+k-j) \phi_{x q}+i(m-l) \phi_{y q}} \operatorname{SEN}\left(\phi_{x q}\right) h_{q_{j k l m}}\left(2 I_{y}\right)^{(l+m) / 2}\left(2 I_{x}\right)^{(j+k-2) / 2} e^{i\left[(1-j+k)\left(2 \pi \nu_{x} N+\psi_{x_{1}}\right)+(m-l)\left(2 \pi \nu_{y} N+\psi_{y_{1}}\right)\right]} .
$$

The complex conjugate of each monomial of this summation is also contained in the summation as can be seen by applying again Eq. (A9). Therefore $\chi(N)$ finally takes the form of Eq. (5). QED.

\section{APPENDIX B: LOCAL RESONANCE TERMS IN THE PRESENCE OF AN AC DIPOLE}

The Hamiltonian resonance driving terms in the presence of an ac dipole are given by [7]

$$
h_{j k 00}^{\prime}=h_{j k 00}^{<}+h_{j k 00}^{>} e^{i(k-j+1) 2 \pi Q_{-}},
$$

where $h_{j k 00}^{<}$contains the contributions to $h_{j k 00}$ of all the elements placed before the ac dipole and $h_{j k 00}^{>}$contains those of the elements placed after. $Q_{-}$is the difference between the driving tune and the betatron tune. When $Q_{-}$ tends to zero, $h_{j k 00}^{\prime}$ tends to $h_{j k 00}$. Equation (1) can be used to approximately describe the motion in the presence of an ac dipole if the $f_{j k l m}$ are replaced by $f_{j k l m}^{\prime}$, the tunes are replaced by the driving tunes, the excitation has been ramped adiabatically, and the tunes are close to the resonance $Q_{D}=Q_{x}$. Under these assumptions we can proceed with the same derivations as in the previous section taking into account Eq. (B1). Ignoring the case when the three BPMs used contain the ac dipole in between them there are only two possibilities: (1) all the local sources are included in $h_{j k 00}^{<}$and (2) all the local sources are included in $h_{j k 00}^{>}$ and will be multiplied by $e^{i(k-j+1) 2 \pi Q_{-}}$as Eq. (B1) states. It is straightforward to conclude that in the first case $\chi_{j k 00}^{\prime}=$ $\chi_{j k 00}$ and in the second case $\chi_{j k 00}^{\prime}=\chi_{j k 00} e^{i(k-j+1) 2 \pi Q_{-}}$. Therefore under the above assumptions the local resonance terms measured from free oscillation and the local terms measured using an ac dipole have the same amplitude.

[1] R. Bartolini and F. Schimdt, Part. Accel. 59, 93 (1998).

[2] R. Tomás, Ph.D. thesis, University of Valencia, 2003.

[3] A. Franchi, T. Beier, M. Kirk, P. Moritz, G. Rumolo, and R. Tomás, in Proceedings of the 2004 European Particle Accelerator Conference, Lucerne, Switzerland (EPS-AG, Lucerne, 2004), http://accelconf.web.cern.ch/accelconf/ e04/default.htm.

[4] R. Tomás, W. Fischer, A. Jain, Y. Luo, and F. Pilat, in Proceedings of the 2004 European Particle Accelerator Conference, Lucerne, Switzerland (Ref. [3]).

[5] W. Fisher, F. Schmidt, and R. Tomás, in Proceedings of the 2003 Particle Accelerator Conference, Portland, OR (IEEE, Piscataway, NJ, 2003).

[6] R. Calaga and R. Tomás, Phys. Rev. ST Accel. Beams 7, 042801 (2004).

[7] R. Tomás, Phys. Rev. ST Accel. Beams 5, 054001 (2002). 